\title{
Single-Electron Temporal Behavior in the Gun Region of the TecnaiFemto UEM
}

Wyatt Curtis ${ }^{1}$ and David Flannigan ${ }^{2}$

${ }^{1}$ University of Minnesota, Minneapolis, Minnesota, United States, ${ }^{2}$ Department of Chemical Engineering and Materials Science, University of Minnesota, Twin Cities, United States

Realization of simultaneous Angstrom-femtosecond ( $\AA$-fs) resolution in ultrafast electron microscopy (UEM) has remained an enduring goal for the field. In a minimally-modified TEM, high spatiotemporal resolutions are attainable in the low bunch-charge regime, where deleterious space-charge interactions are mitigated. In this regime, experimental evidence suggests the ultimate temporal resolution approaches the laser-limited value. ${ }^{1}$ Thus, there is much interest in operating in the so-called single-electron regime, where space-charge effects within the packets are absent. Despite this, statistical temporal broadening can still occur. For example, mismatch between photon energy and cathode work function produces a distribution of photoelectron initial kinetic energies leading to a temporal broadening that is independent of space-charge effects. ${ }^{3-5}$ Further, electron emission statistically following a Gaussian temporal profile will experience time-of-flight chromatic aberrations during acceleration within a constant electric field, though the calculated magnitude of broadening seems to agree poorly with experimental observations. ${ }^{1,6}$ This may be due to specific differences in operating conditions despite the fundamental broadening source still being present. Additionally, studies of Wehnelt biasing have shown that tip effects leading to packet-duration broadening begin to dominate in the high-bias regime. ${ }^{2,7}$ Though potentially discouraging, these findings indicate an opportunity to explore easily-controlled and easily-modified parameters and hardware for optimizing temporal response in the single-electron regime. Indeed, no systematic study of the single-electron temporal response in the gun region of a minimallymodified Thermo Fisher/FEI TecnaiFemto UEM has been done, particularly focusing on easily-controlled space-charge-independent factors (e.g., initial electron kinetic energy, emission spot size, and Wehnelt aperture diameter).

Here, we conduct systematic simulations of single-electron packet durations in the electron-gun region of a Thermo Fisher/FEI TecnaiFemto UEM. We conducted particle tracing simulations with the General Particle Tracer software package and using field maps generated from the precise TecnaiFemto gun architecture with the Poisson Superfish code. ${ }^{8,9}$ As per common Femto operating conditions, the basic gun configuration consisted of an unbiased Wehnelt triode with an aperture diameter Dw and a 180- $\mu$ m diameter truncated LaB6 cathode set-back from the aperture by $350 \mu \mathrm{m}$. Single-electron packets were simulated using a set of noninteracting particles (for reasons of computation efficiency only) emitted from a Gaussian spot with an initial temporal duration of $300 \mathrm{fs}(\mathrm{fwhm})$. Here, three initial kinetic energies were tested: $0.10 \mathrm{eV}, 1.76 \mathrm{eV}$, and $2.40 \mathrm{eV}$. The $0.1-\mathrm{eV}$ and 2.4-eV energies were chosen in order to test the extremes (assuming a photon energy $\mathrm{hv}=4.8 \mathrm{eV}$ and a LaB6 work function $\varphi=2.4 \mathrm{eV}$ ), while $1.76 \mathrm{eV}$ was derived as the average initial energy using a free-electron model with a step potential and approximating photoemission by shifting the electronic distribution by hv. ${ }^{10,11}$ Momentum was distributed along a profile varying as the cosine of the angle between the wave vector and the normal to the surface, and pulse duration was calculated $2.5 \mathrm{~ns}$ after photoexcitation. For each of the energies, the packet duration as a function of emission spot size (i.e., UV probe-laser spot size on the cathode) was simulated for two Wehnelt apertures: a standard 0.7-mm diameter aperture and a larger 1.0-mm diameter aperture. Significant differences for this simple modification were found (Figure 1). For the standard aperture, only the initial electron kinetic energy impacts the duration and, for all energies tested, remains constant out to $180 \mu \mathrm{m}$. However, a strong dependence on emission spot size was found for the larger 1.0-mm aperture. In addition to subtle differences, three behaviorsin particular are worth noting. First, the duration for all energies tested are near the laser limit for spot sizes smaller than $\sim 20$ 
$\mu \mathrm{m}$ for the larger aperture. Second, for this larger aperture, the packets quickly diverge to longer durations for higher-energy electrons with increasing emission spot sizes beyond $20 \mu \mathrm{m}$. At the largest emission-spot size, both of the higher energies show durations of $1.2 \mathrm{ps}$ to $1.5 \mathrm{ps}$ (from an initial 300-fs laser pulse). The duration for these energies drops with smaller emission spot size (e.g., an 80- $\mu \mathrm{m}$ emission spot shows $\sim 1$ psfwhm). Third, despite the differing energies, the higher-energy electrons show the same temporal duration up to an emission spot size of $\sim 45 \mu \mathrm{m}$ before diverging in a perhaps predictable manner. These behaviors are explained as being due to differing trajectories taken by electrons emitted from different points in the spot, with larger spots generally giving a larger range of trajectories and thus ultimate (statistical) temporal durations. More specifically, electrons emitted from the emission-spot edge, as well as those emitted at angles not parallel to the optic axis, experience extended tip-region dwell time and stretched durations. Finally, the non-linear behavior of the higher-energy electrons for the $1.0 \mathrm{~mm}$ aperture suggest a threshold behavior may be present, suggesting a possible dependence on Wehnelt-aperture diameter. These simulations illustrate simple, straightforward ways (e.g., initial kinetic energy, laser-spot size, and Wehnelt-aperture diameter) for optimizing single-electron packet duration in the minimally-modifiedTecnaiFemto UEM and potentially in other such systems. ${ }^{12}$
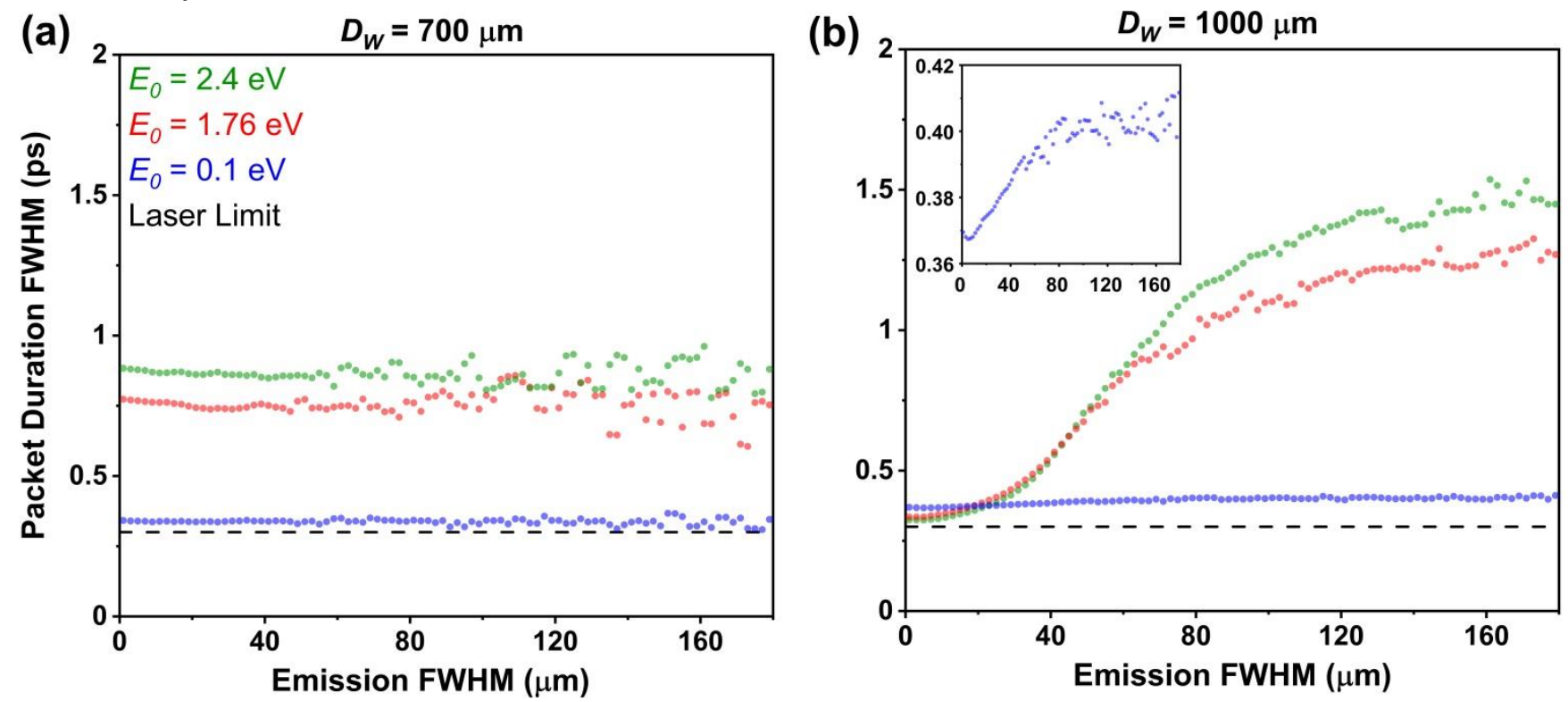

Figure 1. Dependence of photoelectron packet duration on the emission spot size. Simulations were performed at Wehnelt aperture diameters ( $\mathrm{Dw}$ ) of (a) 0.7- $\mathrm{mm}$ and (b) 1.0- $\mathrm{mm}$. For each aperture diameter, three initial electron kinetic energies were tested: $0.1 \mathrm{eV}$ (blue), $1.76 \mathrm{eV}$ (red), and $2.4 \mathrm{eV}$ (green). The black dashed line indicates the laser-limited packet duration assuming photoexcitation with a $300 \mathrm{fs}$ ( $\mathrm{fwhm}$ ) laser. The inset in panel (b) is a rescaled plot of the $0.1 \mathrm{eV}$ dataset shown in the corresponding panel.

\section{References}

1. Plemmons, D. A.; Flannigan, D. J. Chem. Phys. Lett. 2017, 683, 186-192.

2. Piazza, L.; et al. Chem. Phys. 2013, 423, 79-84.

3. Aidelsburger, M.; et al. Proc. Natl. Acad. Sci. U.S.A. 2010, 107, 19714-19719.

4. Baum, P.; Zewail, A. H. Proc. Natl. Acad. Sci. U.S.A. 2006, 103, 16105-16110.

5. Baum, P. Chem. Phys. 2013, 423, 55-61.

6. Aseyev, S. A.; et al. J. Exp. Theor. Phys.2019, 128, 379-383.

7. Shaozheng, J.; et al. Struct. Dyn.2017, 4, 054303. 
8. De Loos, M. J.; Van der Geer, S. B. General Particle Tracer: A New 3D Code for Accelerator and Beamline Design. Proceedings of the European Particle Accelerator Conference, Sitges (Barcelona), Spain, June 10-14, 1996, p. 1241.

9. Halbach, K.; Holsinger, R. F. Part. Accel.1976, 7, 213-222.

10. Mogren, S.; Reifenberger, R. Surf. Sci. 1991, 254, 169-181.

11. Mogren, S.; Reifenberger, R. Surf. Sci. 1987, 186, 232-246.

12. We thank Erik Kieft for assistance with modeling the TecnaiFemto architecture and ensuring accurate electrostatic field maps were generated. This material is based on work supported by the U.S. Department of Energy, Office of Science, Office of Basic Energy Sciences under Award No. DE-SC0018204. This material is based upon work supported by the National Science Foundation Graduate Research Fellowship Program under Grant No. DGE-1839286. Acknowledgment is made to the Donors of the American Chemical Society Petroleum Research Fund for partial support of this research. 